\title{
HEMORRAGIA ADRENAL BILATERAL COM TROMBOSE DA VEIA RENAL DIREITA E VEIA CAVA INFERIOR EM UM RECÉM-NASCIDO*
}

\author{
Severino Aires de Araújo Neto', Rodrigo Tranquilini Rezende ${ }^{2}$, Antônio Soares de Souza ${ }^{3}$, \\ José Maria Pereira de Godoy ${ }^{4}$
}

\begin{abstract}
Resumo Os autores apresentam um caso de um recém-nascido com um tipo raro de associação de hemorragia adrenal bilateral com trombose de veia renal direita e de veia cava inferior, em que os exames de ultra-sonografia e tomografia computadorizada exerceram papel crucial no estabelecimento do diagnóstico, orientação da conduta e seguimento do paciente.

Unitermos: Trombose; Glândula adrenal; Hemorragia; Neonato; Ultra-sonografia; Tomografia computadorizada.
\end{abstract}
Abstract Bilateral adrenal hemorrhage associated with right renal vein and inferior vena cava thrombosis in a new- born.
We report a rare case of coexisting bilateral adrenal hemorrhage, right renal vein and inferior vena cava throm- bosis in a neonate. Imaging studies played an important role in the diagnosis, management, and follow-up of this patient.
Key words: Thrombosis; Adrenal glands; Hemorrhage; Neonate; Ultrasound; Computed tomography.

\section{INTRODUÇÃO}

A trombose da veia renal e a hemorragia adrenal são duas entidades distintas, cuja associação tem sido relatada em recém-nascidos ${ }^{(1-12)}$.

A hemorragia adrenal é uma das causas menos freqüentes de massa abdominal em recém-nascidos, e a hidronefrose e o rim multicístico displásico as mais comuns ${ }^{(1,13)}$. A veia renal é o principal sítio de trombose nesta faixa etária ${ }^{(14)}$. A veia cava inferior é acometida com menor frequiência. Complicações do quadro clínico podem decorrer de falência renal e hipertensão $0^{(10)}$.

Neste artigo é apresentada uma associação rara de hemorragia adrenal com trombose da veia renal direita e trombose de veia cava inferior em um recém-nascido, no qual os estudos imaginológicos exerceram

* Trabalho realizado no Hospital de Base da Faculdade de Medicina de São José do Rio Preto (Famerp), São José do Rio Preto, SP.

1. Membro Titular do Colégio Brasileiro de Radiologia e Diagnóstico por Imagem (CBR), Médico do Centro de Atendimento Integrado da Saúde da Mulher (CAISM) da Universidade Estadual de Campinas (Unicamp).

2. Membro Titular do CBR, Serviço de Radiologia da Santa Casa de Misericórdia de Mococa, SP.

3. Professor e Chefe do Serviço de Radiologia do Hospital de Base da Funfarme/Famerp.

4. Professor da Disciplina de Cirurgia Vascular do Hospital de Base da Funfarme/Famerp.

Endereço para correspondência: Dr. Severino Aires de Araújo Neto. Rua José Ferreira Lameirão, 77, ap. 32. Campinas, SP, 13070-380. E-mail: severinoaires@hotmail.com

Recebido para publicação em 27/9/2002. Aceito, após revisão, em 22/4/2003. papel decisivo no diagnóstico, orientação da conduta e seguimento do paciente.

\section{RELATO DO CASO}

Recém-nascido do sexo feminino, prétermo, nascido em outra instituição, via incisão cesariana, na 37ª semana de uma gestação complicada por pré-eclâmpsia. Não havia história de diabetes materno. $\mathrm{O}$ peso ao nascer foi de $3.750 \mathrm{~g}$, com grau Apgar de 4 e 7 (1 e 5 minutos).

O recém-nascido foi transferido, no mesmo dia, à unidade de terapia intensiva neonatal do Hospital de Base da Faculdade de Medicina de São José do Rio Preto, SP, frente a um quadro de insuficiência respiratória. Ventilação mecânica e cateterização umbilical foram prontamente instauradas. A criança evoluiu com icterícia, hematúria macroscópica e distensão abdominal, sendo então palpada uma massa no flanco direito. Exames laboratoriais de admissão revelaram anemia discreta, hiperbilirrubinemia (à custa de fração indireta), proteinúria e hematúria.

Foi solicitada tomografia computadorizada (TC) do abdome no segundo dia de vida, para descartar a possibilidade de massa neoplásica. Este exame demonstrou, na topografia das adrenais, bilateralmente, massas hipodensas que não se realçaram com o meio de contraste iodado endovenoso não-iônico (Figura 1). Não se obser- vou, também, realce intraluminal pelo meio de contraste da veia renal direita e da veia cava inferior infra-hepática, as quais apresentaram-se dilatadas por material hipodenso intraluminal, representando trombos (Figuras 2A e 2B). O rim direito estava aumentado de volume. Após a infusão do meio de contraste iodado endovenoso, houve realce da cápsula, mas não do parênquima desse rim (Figura $2 \mathrm{~B}$ ). O rim esquerdo não apresentou alterações.

Diante do quadro clínico e da evidência de trombos na veia renal direita e na veia cava inferior, chegou-se a um diagnóstico de hemorragia adrenal bilateral. No dia

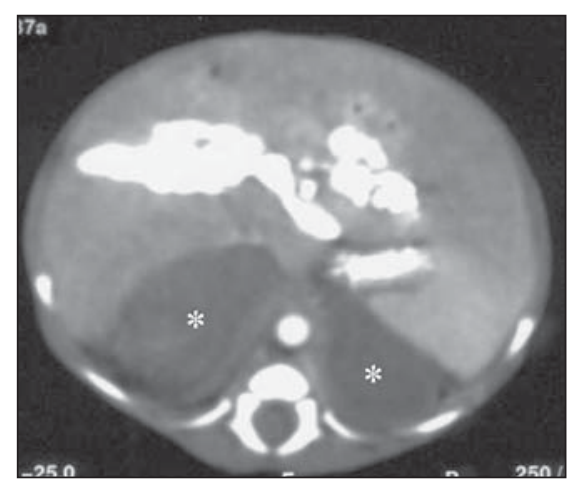

Figura 1. TC após administração de meio de contraste iodado endovenoso, em um nível superior aos rins, demonstrando massas hipodensas bilaterais (asteriscos), que não se realçam com o meio de contraste, representando a hemorragia adrenal. 0 meio de contraste é visto preenchendo o leito vascular hepático, devido ao posicionamento do cateter na topografia do ducto venoso. 
seguinte (terceiro dia de vida), em uma ultra-sonografia (US) abdominal, a hemorragia adrenal apresentava-se como massas predominantemente anecóicas (Figuras 3A e 3B). O rim direito estava aumentado e possuía ecotextura heterogênea (Figura 3A). Ao estudo com Doppler havia redução do sinal de fluxo desse rim (Figura 4A) em relação ao rim contralateral (Figura 4B). Trombos hipoecóicos foram observados na veia renal direita (Figura 5A) e na veia cava inferior (Figura 5B), que estavam desprovidas de sinal de fluxo.

A conduta foi conservadora, consistindo de antibioticoterapia parenteral (por causa de pneumonia nosocomial) e anticoagulação com heparina, de início, e com anticoagulantes orais, posteriormente.

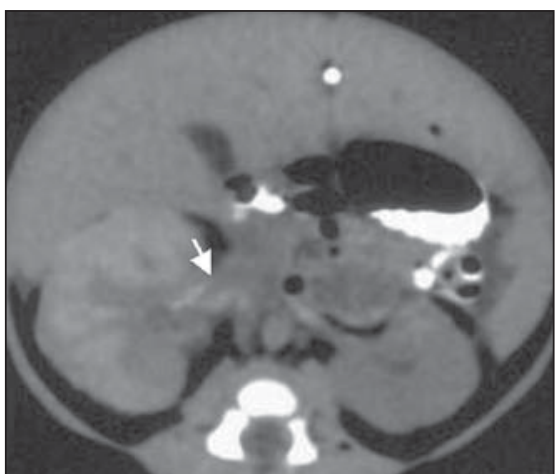

A

Figura 2. TC pré (A) e pós-meio de contraste iodado endovenoso (B), no nível dos rins. Trombose venosa. Em A, a veia renal direita (seta branca) está dilatada por trombo predominantemente hipodenso, com estrias hiperdensas de permeio. 0 trombo na veia cava inferior $(\mathbf{B})$ impede seu realce pelo meio de contraste (seta preta). 0 rim direito está aumentado de volume, entremeando áreas de hiperdensidade, que correspondem às pirâmides, interpretadas como áreas de infartos hemorrágicos (A). 0 parênquima renal não apresenta realce pelo contraste (B). Apenas um fino halo de realce (setas brancas em B), representando a cápsula renal, é visualizado. As hemorragias adrenais estão marcadas com asteriscos em $\mathbf{B}$.

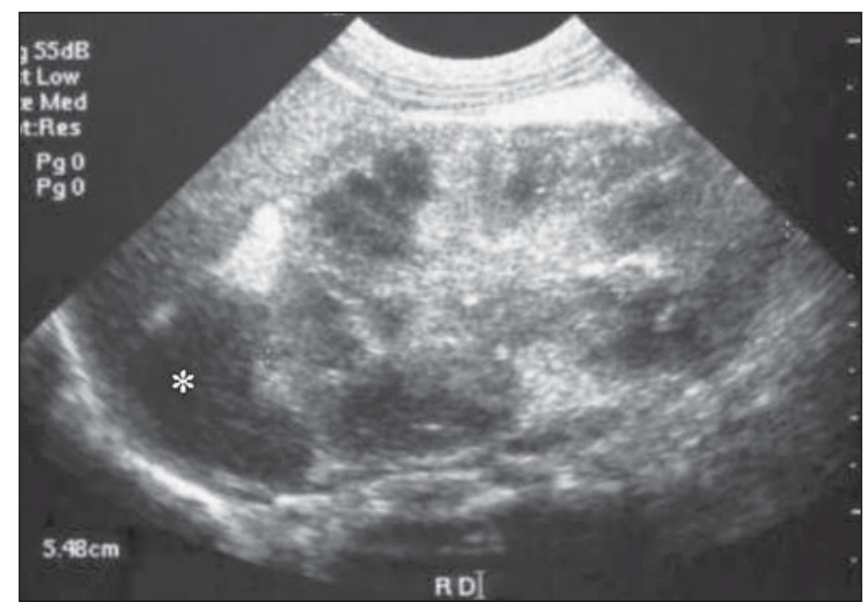

A

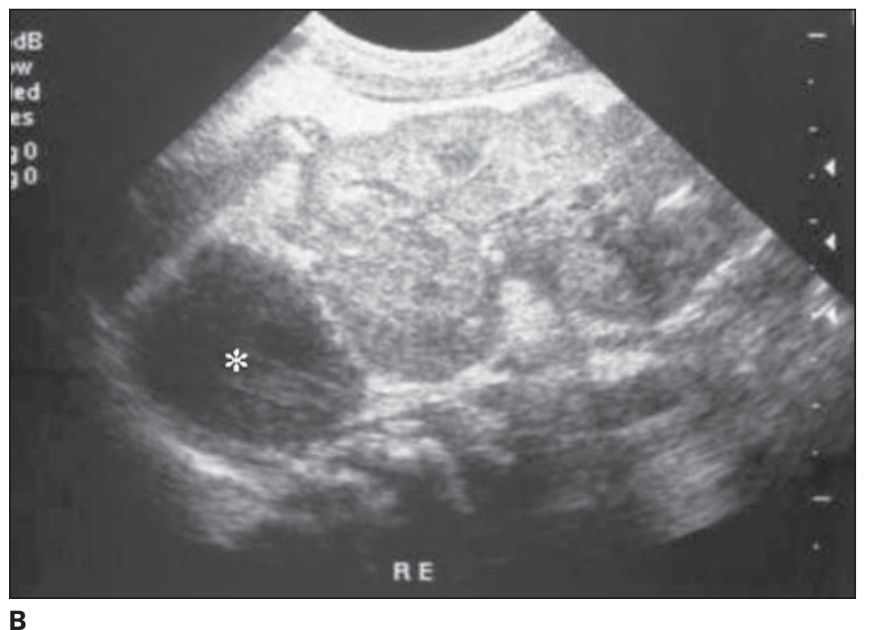

B

Figura 3. US no plano longitudinal dos rins direito $(\mathbf{A})$ e esquerdo (B). As hemorragias adrenais apresentam-se como massas anecóicas adjacentes ao pólo superior de cada rim (asteriscos). Devido à trombose da veia renal à direita, o rim direito (A) encontra-se aumentado de volume, com ecotextura heterogênea do seu parênquima e relativa hipoecogenicidade de suas pirâmides.

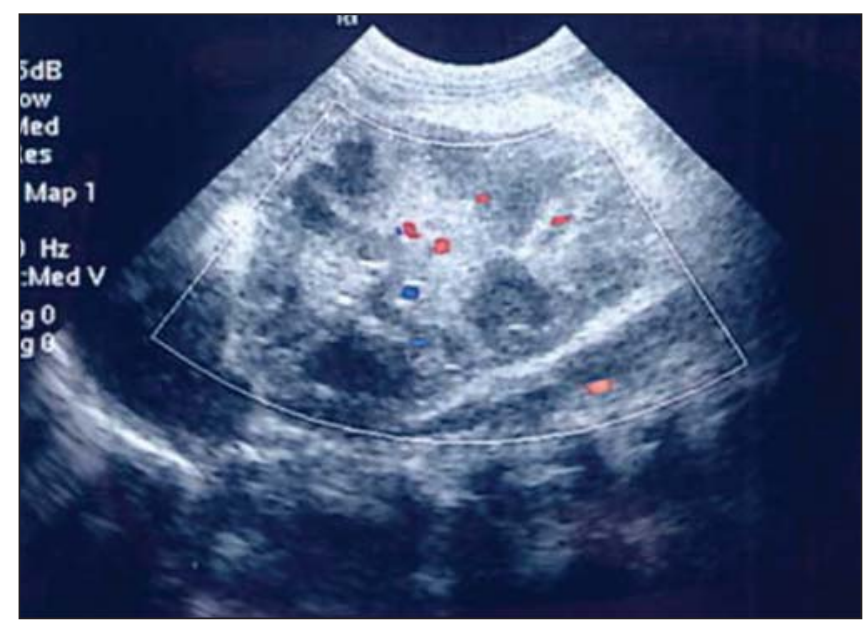

A

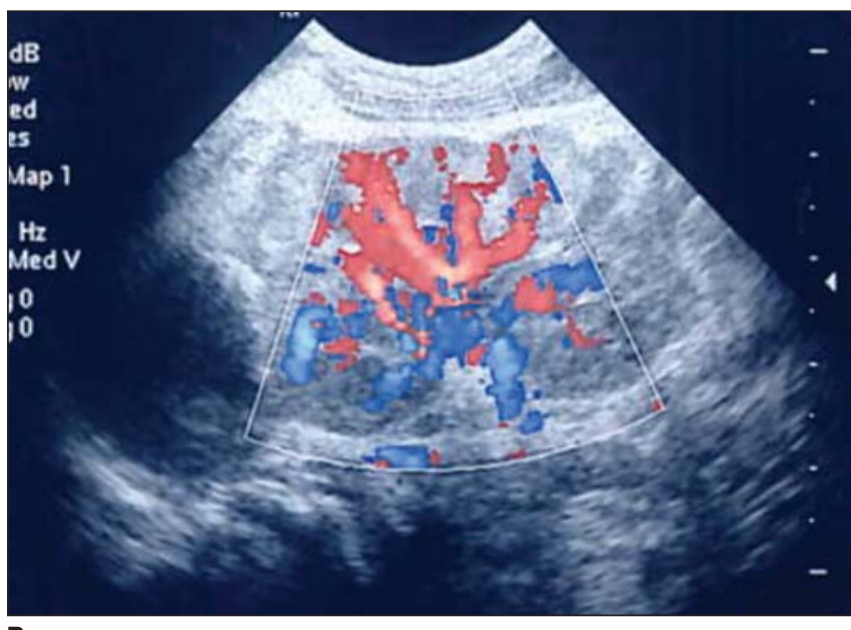

Figura 4. US Doppler colorido no plano longitudinal dos rins. Rim direito (A) com acentuada redução do fluxo. Comparação com o rim esquerdo (B). 

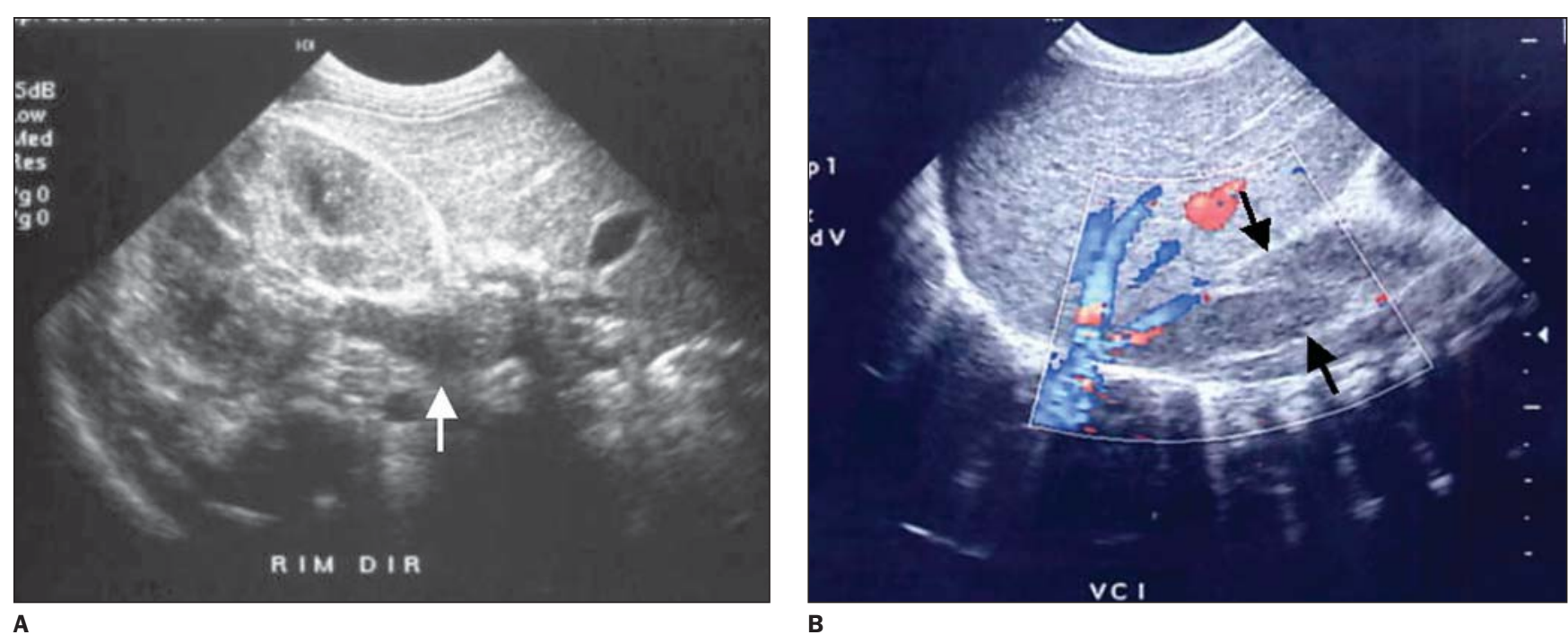

A

Figura 5. US modo B. A: Em plano transverso, observa-se o trombo hipoecóico dilatando a veia renal direita (seta branca). B: Estudo com Doppler colorido, em plano longitudinal, demonstrando a trombose na veia cava inferior infra-hepática (entre setas pretas), a qual se encontra desprovida de sinal de fluxo. Observar que as veias hepáticas e a veia cava inferior supra-hepática estão pérvias.

Duas semanas após a admissão, numa cintilografia renal, havia perda da função do rim direito. Na evolução, a criança desenvolveu síndrome de Addison e hipertensão arterial sistêmica, controladas clinicamente. Aos três meses de vida, numa US de seguimento, havia resolução da hemorragia adrenal e atrofia do rim direito (Figura 6).

\section{DISCUSSÃO}

A associação de hemorragia adrenal com a trombose venosa profunda é evento raro, ocorrendo classicamente no período neonatal. Diferentes combinações têm sido descritas $^{(1-12)}$, das quais, a mais comum é a associação da trombose da veia renal com a hemorragia adrenal à esquerda, com ou sem trombose da veia cava inferior.

A hemorragia adrenal é causa pouco freqüente de massa abdominal ${ }^{(1,13)}$. Trauma no parto, sepse, hipoxemia, diabetes materno e trombose da veia renal são fatores associados. Sua patogenia é controversa $^{(5,8)}$. No recém-nascido, o tamanho da adrenal relativo ao rim e sua vascularização são maiores do que no adulto. Segundo alguns autores, isto reduziria a resistência do leito vascular da glândula, tornando-a mais suscetível às variações de pressão intra-abdominal ${ }^{(4,9)}$. Especula-se também que uma compressão entre o fígado e o rim direito explicaria a maior frequiência

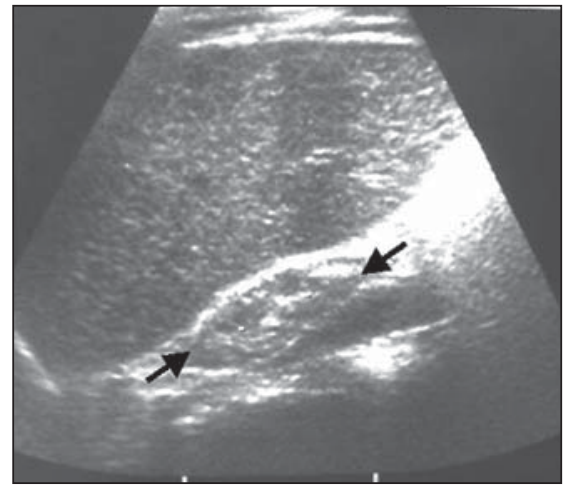

Figura 6. US de seguimento no plano anatômico coronal oblíquo demonstrando atrofia do rim direito (entre setas) e a veia cava inferior pérvia, anecóica, medialmente ao rim.

(70\%) com que a hemorragia adrenal ocorre deste lado $^{(4,6)}$. O ingurgitamento passivo da glândula ou a isquemia (secundários, respectivamente, à trombose venosa e à hipóxia, por exemplo), também podem estar envolvidos ${ }^{(2)}$.

A hemorragia adrenal pode ocorrer intra-útero, sendo alguns casos diagnosticados por US obstétrica ${ }^{(\mathbf{1}, \mathbf{9})}$. Apesar de ocorrer com maior freqüência do lado direito, sua associação com trombose da veia renal é mais comum do lado esquerdo, provavelmente pelo fato de a veia adrenal ser tributária da veia renal desse lado, permitindo que o trombo propague-se da veia adrenal diretamente para a veia renal esquerda e até para a veia cava inferior. No lado di- reito, por sua vez, a veia renal e a veia adrenal desembocam na veia cava inferior separadamente $e^{(1,2)}$.

A apresentação clínica usual da hemorragia adrenal consiste de massa no flanco,

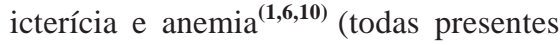
neste caso). A hematúria sugere associação com trombose da veia renal ${ }^{(2)}$. Complicações consistem na hemorragia incontrolável, formação de abscesso e trombose da veia renal com hipertensão (devido à disfunção renal, como ocorrido no caso em discussão). A insuficiência adrenal é rara, mesmo em casos com hemorragia adrenal bilateral ${ }^{(\mathbf{1 0})}$. Neste relato, a criança desenvolveu síndrome de Addison.

A trombose venosa é relativamente incomum em recém-nascidos ${ }^{(15)} \mathrm{e}$, em crianças, $70 \%$ dos casos acontecem na veia renal, usualmente unilateral e mais comumente à esquerda ${ }^{(\mathbf{1 3})}$. Fatores predisponentes incluem hemoconcentração no curso de desidratação importante, sepse, cateterismo vascular e diabetes materno ${ }^{(6,8,9,94,15)}$, podendo, como discutido acima, constituir uma complicação de hemorragia adrenal.

$\mathrm{Na}$ trombose da veia renal, quando o acometimento é bilateral, o recém-nascido apresenta insuficiência renal. No envolvimento unilateral podem estar presentes hematúria, proteinúria, hipertensão (o rim comprometido responde com aumento da produção de renina), anemia (todas observadas neste caso) e trombocitopenia. 
A evolução do trombo é variável, ocorrendo desde o desaparecimento completo em poucos dias até calcificação e estenose venosa persistente ${ }^{(9)}$. O rim pode recuperar a função se a circulação colateral for adequada ou houver recanalização precoce da veia renal. Caso contrário, o órgão sofrerá atrofia, com perda funcional.

A terapia é de suporte e deve incluir remoção dos fatores precipitantes e tratamento da eventual disfunção renal. O uso de trombolíticos pode ser benéfico. Recomenda-se o acompanhamento a longo prazo pelo risco de desenvolvimento de disfunção renal e hipertensão ${ }^{(\mathbf{1 , 1 0})}$.

É provável que a pré-eclâmpsia materna e/ou insuficiência respiratória pós-natal, levando à hipoxemia, tenham sido os fatores predisponentes para a hemorragia adrenal neste caso. A trombose venosa pode, então, ter ocorrido como uma complicação da hemorragia adrenal ou ter sido um fator concomitante, independente, possivelmente relacionada ao cateterismo umbilical, visto que grande parte das tromboses venosas neonatais estão relacionados a cateteres vasculares centrais ${ }^{(\mathbf{1 4 - 1 6 )}}$. Não se pode descartar, porém, a possibilidade de o evento inicial ter sido a trombose venosa, desencadeando a hemorragia adrenal por ingurgitamento do leito vascular da glândula, como explicado acima.

O fenômeno trombótico pode eclodir no leito renal, progredindo, posteriormente, para a veia cava inferior e veias adrenais, ou, ao contrário, pode iniciar-se na veia cava inferior e progredir para o rim $^{(\mathbf{1})}$. É possível que este último tenha sido o mecanismo de progressão ocorrido no recémnascido do presente relato, conseqüente a uma complicação de cateterismo venoso umbilical ${ }^{(14-16)}$. Os distúrbios hemodinâmicos proporcionados pela pré-eclâmpsia e a hipóxia pós-natal também podem ter contribuído na patogênese da trombose da veia renal. Nenhum outro fator de risco descrito na literatura foi identificado. Contudo, como o parto do bebê se deu em outra instituição, as condições perinatais não foram completamente esclarecidas e alguns dados essenciais podem estar sendo negligenciados.

Dois aspectos conferem uma peculiaridade especial ao caso aqui apresentado e devem ser enfatizados: a bilateralidade da hemorragia adrenal - menos freqüente do que sua ocorrência unilateral - e sua associação com a trombose da veia renal do lado direito ${ }^{(\mathbf{2 , 4 , 6 )}}$. Do nosso conhecimento, somente seis casos de associação de hemorragia adrenal com trombose da veia renal à direita foram descritos ${ }^{(\mathbf{2 , 4}, 11,12)}$, dentre as quais, a mais complexa foi uma combinação de trombose da veia renal e hemorragia adrenal, ambas bilaterais, associada a trombose de veia cava inferior ${ }^{(4)}$.

$\mathrm{Na}$ abordagem de um recém-nascido com suspeita de hemorragia adrenal e/ou de trombose da veia renal, o uso dos exames por imagem deve ser criterioso. Uma abordagem imaginológica inadequada pode culminar em atraso no diagnóstico e em intervenções terapêuticas desnecessárias. Por exemplo, num caso ocorrido em $1976^{(6)}$, uma associação de hemorragia adrenal esquerda com trombose da veia renal à direita apresentou-se, à urografia excretora, como uma massa no flanco esquerdo, associada a exclusão renal direita. Os exames de medicina nuclear e a pielografia ascendente não esclareceram o diagnóstico. A US e a TC não foram realizadas e o recém-nascido teve de ser submetido a uma exploração cirúrgica, na qual constatou-se a natureza hemorrágica da massa adrenal. Ainda no mesmo artigo é descrito outro caso, ocorrido mais de um ano depois, em que houve associação de hemorragia adrenal e trombose da veia renal ipsilaterais. Neste último, a US estabeleceu o diagnóstico, direcionando à terapia clínica adequada.

Portanto, diante da suspeita de hemorragia adrenal e/ou de trombose da veia renal, a US deve constituir o método de imagem inicial de escolha, pois tem acurácia semelhante à da TC, não envolve radiação ionizante, tem menor custo e é mais prática (não requer sedação e pode ser realizada à beira do leito) ${ }^{(3,9)}$. Além disso, no recém-nascido, a escassez de gordura intraabdominal dificulta o estudo tomográfico. Deve-se lembrar também que o uso de meio de contraste iodado endovenoso não é desprovido de riscos, especialmente em neonatos nos primeiros dias de vida, quando a capacidade de filtração glomerular é de cerca de $20 \%$ em relação ao adulto $^{(17)}$. Se for indispensável o uso do meio de contraste iodado endovenoso, é aconselhável optar pelas fórmulas não-iônicas. Contudo, em boa parte dos casos, a TC é dispensável, podendo vir a ser solicitada se não for levantada inicialmente a suspeita de hemorragia adrenal pela US.

Na US, a aparência da hemorragia adrenal varia de acordo com o tempo de evolução, de ecogênica para anecogênica ${ }^{(3,7,9)}$. O principal diagnóstico diferencial da hemorragia adrenal é o neuroblastoma neonatal. Uma involução da massa demonstrada por US seriada com liquefação central e resolução dentro de até seis semanas, e a dosagem normal de catecolaminas urinárias afastarão a suspeita de natureza neoplásica da lesão ${ }^{(4)}$.

O estudo por ressonância magnética (RM) pode também ajudar nessa diferenciação, sendo que a hemorragia aparece com sinal intenso tanto na ponderação em $\mathrm{T} 1$ quanto em $\mathrm{T} 2^{(3)}$.

$\mathrm{Na}$ trombose da veia renal, a associação com o Doppler é importante ${ }^{(\mathbf{1 0})}$. O trombo pode aparecer hipo ou hiperecóico. A sua visualização direta pela US na veia renal é especialmente rara em recém-nascidos ${ }^{(2)}$. Por exemplo, dentre os 19 casos revisados de associação de hemorragia adrenal com trombose da veia renal examinados por $\mathrm{US}^{(\mathbf{1 - 1 1})}$, apenas um autor ${ }^{(\mathbf{1 0})}$ descreveu este achado. $\mathrm{O}$ achado mais freqüente é o de um rim afetado com aumento de volume e faixas de hiperecogenicidade.

Os exames com radionuclídeos são muito sensíveis na demonstração da redu-

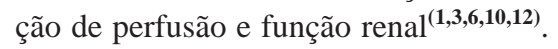

Os achados à TC incluem, na hemorragia adrenal, massa adrenal hipodensa, e, na trombose da veia renal, ausência de realce renal pelo contraste ou persistência do realce, opacificação retardada ou ausente do sistema coletor, trombo dilatando a veia renal, circulação colateral perirrenal e espessamento da fáscia de Gerotta ${ }^{(4)}$.

Nos dias atuais, a urografia excretora traz pouca informação adicional. Pode-se observar efeito de massa da hemorragia adrenal sobre o rim, com desvio inferior deste. Na trombose da veia renal, observase exclusão do rim afetado ${ }^{(6,9)}$. Calcificações residuais distróficas na glândula podem aparecer já na primeira semana após o diagnóstico ${ }^{(8,10)}$.

Apesar do exuberante quadro apresentado no presente relato, o diagnóstico não 
foi suspeitado clinicamente, provavelmente por causa da raridade da doença, bem como pela gravidade e rápida evolução do quadro. Uma massa abdominal palpável chamou mais a atenção do que os demais indícios clínicos, induzindo a uma suspeita inicial de neoplasia, provavelmente um neuroblastoma. Estas dificuldades podem explicar a abordagem imaginológica que expôs o paciente precipitadamente aos riscos da radiação por TC e da administração de meio de contraste iodado endovenoso, antes do exame de US, que, no caso em questão, elucidaria de modo satisfatório o diagnóstico.

\section{CONCLUSÃO}

A associação de hemorragia adrenal com trombose da veia renal é um evento neonatal raro, porém facilmente diagnosticado por métodos de imagem não-invasivos e de baixo custo.

O diagnóstico precoce é essencial para o sucesso terapêutico. A US com estudo por Doppler é o exame inicial de escolha, pois é prático, acurado e de baixo custo, não envolvendo os riscos da radiação ionizante e do meio de contraste iodado endovenoso. O estudo ultra-sonográfico é útil também para o acompanhamento da resposta à terapia, que nos casos não complicados é essencialmente clínica. A medicina nuclear serve para avaliar a função renal. A TC e a RM são complementares e podem trazer dados adicionais importantes apenas em casos selecionados.

\section{REFERÊNCIAS}

1. Bennett WG, Wood BP. Radiological case of the month. Left renal vein thrombosis and left adrenal hemorrhage. Am J Dis Child 1991;145:1299-300.

2. Bowen A, Smazal SF Jr. Ultrasound of coexisting right renal vein thrombosis and adrenal hemorrhage in a newborn. J Clin Ultrasound 1981;9:511-3.

3. Brill PW, Jagannath A, Winchester P, Markisz JA, Zirinsky K. Adrenal hemorrhage and renal vein thrombosis in the newborn: MR imaging. Radiology 1989;170(1 Pt 1):95-8.

4. Demirci A, Selcuk MB, Yazicioglu I. Bilateral adrenal hemorrhage associated with bilateral renal vein and vena cava thrombosis. Pediatr Radiol 1991;21:130.

5. Koch KJ, Cory DA. Simultaneous renal vein thrombosis and bilateral adrenal hemorrhage: MR demonstration. J Comput Assist Tomogr 1986;10:6813.

6. Lebowitz JM, Belman AB. Simultaneous idiopathic adrenal hemorrhage and renal vein thrombosis in the newborn. J Urol 1983;129:574-6.

7. Levine C. Intestinal obstruction in a neonate with adrenal hemorrhage and renal vein thrombosis. Pediatr Radiol 1989;19:477-8.

8. Orazi C, Fariello G, Malena S, Schingo P, Ferro F, Bagolan P. Renal vein thrombosis and adrenal hemorrhage in the newborn: ultrasound evaluation of
4 cases. J Clin Ultrasound 1993;21:163-9.

9. Tran-Minh VA, Genin G, Pracros JP, Foray P, Bourgeois J. Coexisting calcified inferior vena cava thrombus and adrenal hemorrhage in the neonate: report of three cases. J Clin Ultrasound 1994;22: 103-8.

10. Veiga PA, Springate JE, Brody AS, Cummings JJ, Mosovich L, Feld LG. Coexistence of renal vein thrombosis and adrenal hemorrhage in two newborns. Clin Pediatr (Phila) 1992;31:174-6.

11. Potter BM. Diagnostic ultrasound's role in the pediatric abdomen. Appl Radiol 1979;8:95.

12. Suga K, Hara A, Motoyama K, Ishikawa Y, Kume $\mathrm{N}$, Matsunaga N. Coexisting renal vein thrombosis and bilateral adrenal hemorrhage: renoscintigraphic demonstration. Clin Nucl Med 2000;25:263-7.

13. Buonomo C, Taylor GA, Share JC, Kirks DR. Gastrointestinal tract. In: Kirks DR, Griscom NT, eds. Practical pediatric imaging: diagnostic radiology of infants and children. Philadelphia, PA: LippincottRaven, 1998:836.

14. Bokenkamp A, von Kries R, Nowak-Gottl U, Gobel U, Hoyer PF. Neonatal renal venous thrombosis in Germany between 1992 and 1994: epidemiology, treatment and outcome. Eur J Pediatr 2000;159: 44-8.

15. Mehta S, Connors AF Jr, Danish EH, Grisoni E. Incidence of thrombosis during central venous catheterization of newborns: a prospective study. Pediatr Surg 1992;27:18-22.

16. Edstrom CS, Christensen RD. Evaluation and treatment of thrombosis in the neonatal intensive care unit. Clin Perinatol 2000;27:623-41.

17. Barnewolt CE, Paltiel HJ, Lebowitz RL, Kirks DR Genitourinary tract. In: Kirks DR, Griscom NT, eds. Practical pediatric imaging: diagnostic radiology of infants and children. Philadelphia, PA: LippincottRaven, 1998:1013. 